\title{
Prevalencia de fractura de cadera en adultos mayores institucionalizados en la Provincia Santiago
}

\author{
IRENE VÁSQUEZ(1), CYNTHIA VALENZUELA(1), CAROLA ZAPATA(1), SAMANTA RUZ(1), \\ VALERIA VERONESI(1), VERÓNICA IGLESIAS(2), PAULINA PINO(2)
}

\section{RESUMEN}

El objetivo fue establecer la prevalencia de fractura de cadera en adultos mayores (AM) que residen en instituciones de beneficencia en la provincia de Santiago. Se realizó un estudio transversal, mediante la revisión de 200 fichas clinicas seleccionadas por muestreo aleatorio estratificado de mayores de 60 años. La prevalencia fue de 9,5\%. En la muestra 62,5\% fueron mujeres con un promedio de edad de 81,9+8,8, y un 37,5\% hombres con un promedio de edad de 78,1 + 8,6 En cambio, en los fracturados el promedio de edad fue 82,3 $\pm 9,7$. El principal lugar de caidas fue el baño (26,3\%). La secuela más importante fue la postración la cual anmentó en 2,5\%. Se observó menor promedio peso corporal y un menor promedio de IMC en los fracturados $(p<0,05)$ y el riesgo de tener fracturas fue mayor en quienes presentaban antecedentes previos de fractura (RP 2,57; IC95\% 1,1-5,8). Estos resultados contribuven al conocimiento epidemiológico de este problema en el país, destacando que la existencia de instituciones no registradas posiblemente enmascara lina situación aín más preocupante en términos de equidad en salud. Se requiere profundizar estos estudios para establecer la magnitud y tendencias de la fractura de cadera en los AM no institucionalizados y para obtener una mejor comprensión de los factores de riesgo.

Palabras claves: Fractura de cadera, prevalencia, instituciones de beneficencia, factores de riesgo.

\section{ABSTRACT}

\section{PREVALENCE OF HIP FRACTURE IN ELDERLY}

The aim was to establish the prevalence of hip fracture in the elderly residing in benefic institutions in Santiago city. A cross-sectional study of 200 institutional clinic records of interned $>60$ years selected by proportional stratified random sampling was performed. The prevalence of hip fracture was $9.5 \%$. Women comprised $62.5 \%$ of the sample with ages averaging $81.9+8.8$ whilst $37.5 \%$ were men with a mean age of $78.1+8.6$. Conversely, the mean age of the fractured within the sample was $82.3 \pm 9.7$ years. The main place of occurrence was the bathroom (26.3\%). Prostration was the main sequel, increasing in 2.5\% after the fracture. Mean weight and IMC were lower $(p<0.05)$ in the fractured elders compared with those that did not experience fracture and the risk of fracture was mayor in those who had preview antecedents of fracture (RP 2.57; IC95\% 1.1-5.8). The results contribute to the epidemiological knowledge of this pathology in the

14) Estudiante de $V l^{\circ}$ año de Medicina. Universidad de Chile. irenesolylluviaramixmail.com

(2) Escuela Salud Publica. Facultad de Medicina. Universidad de Chile. 
country though a hidden situation in non-registered institutions should be pointed out as a primary concern related to social and health equity. New studies are required to establish the prevalence in non-institutionalized population and to obtain a better understanding of the risk factors.

Key words: Hip fracture, prevalence, epidemiology, benefic institutions, risk factors.

\section{INTRODUCCIÓN}

La población de adultos mayores en nuestro país cursa por un período de crecimiento sin precedente. Mientras que en 1960 correspondía sólo al 3,8\% de la población nacional, hoy alcanza un 11,4\%. Según el censo de 2002 hay 90 adultos mayores por cada 100 jóvenes menores de 14 años!

Una de las patologías que produce mayor discapacidad en este grupo etáreo es la fractura de cadera ${ }^{2}$. Cambios hormonales, óseos, cognitivos y de alimentación entre otros, predisponen a este trastorno, por lo que ha sido reportada en diferentes paises como la patología más prevalente en dicho grupo ${ }^{3}$. En España, país con características étnicas similares a las de Chile, la fractura de cadera alcanza una prevalencia de 5 por cada 1.000 mayores de 64 años ${ }^{4}$.

En Chile, no existen estudios previos de prevalencia de esta patología, sin embargo, entre 1980 y 1983 se registró un aumento de $16 \%$ en la tasa de egresos hospitalarios por fractura de cadera, pasando de 140 a 162 por 100.000 habitantes ${ }^{4}$, cifra que refleja muy cercanamente la verdadera prevalencia poblacional. La edad y el sexo son factores de riesgo importantes. Mientras a los 50 años la prevalencia es de 7 por cada 100.000 varones $y$ 2 por cada 100.000 mujeres, a los 80 años esta patología aumenta a 475 y 1.200 por cada 100.000 habitantes, respectivamente ${ }^{5}$.

Un estudio de caso-control realizado a partir de egresos hospitalarios de la Región Metropolitana entre 1993 y 1994 , exploró como posibles factores de riesgo las características antropométricas, la utilización de lentes ópticos, el lugar de ocurrencia de caídas, histerectomia, consumo de leche durante el embarazo, y tabaquismo?. Sólo la histerectomía $(\mathrm{OR}=2,0 ; \mathrm{IC} \quad 1,1-3,6 ; \mathrm{p}<0,05)$ y el no consumo de leche $(\mathrm{OR}=1,8 ; 1 \mathrm{C} 1,1-2,9 ; \mathrm{p}<$
0,001) registraron efectos estadísticamente significativos.

Por otra parte, la nueva realidad demográfica determina que un número creciente de adultos mayores residan en instituciones. Del total de adultos mayores del pais, $1 \% \quad(5.000$ aproximadamente) vive en la provincia de Santiago' y de ellos, 44\% reside en hogares de beneficencia, realidad que hasta ahora no ha sido abordada. El presente estudio tiene como objetivo principal determinar la prevalencia de fractura de cadera en ancianos de instituciones de beneficencia de la provincia de Santiago. Secundariamente se estudiarán los factores de riesgo asociados con el fin de contribuir a la elaboración de estrategias preventivas.

\section{MATERIAL Y MÉTODO}

El diseño de este estudio es de tipo transversal. Se consideró un universo de 2.180 adultos mayores, equivalente a los mayores de 60 años que residen en instituciones de beneficencia en la provincia de Santiago a la fecha del estudio (julio 2003). No se incluyó a las instituciones que no estaban acreditadas por sus respectivos Servicios de Salud puesto que no hay registros, y se excluyó a las instituciones privadas para evitar el efecto confundente de la condición socioeconómica. Se utilizó una muestra aleatoria estratificada. Ésta se obtuvo calculando el porcentaje de adultos mayores institucionalizados correspondientes a cada Servicio de Salud. Para ello se consideró el total de instituciones, independiente del tamaño de residentes (Figura 1). Mediante un estudio piloto realizado en una muestra aleatoria $(n=58)$ se evaluó la aplicabilidad y adecuación de una ficha ad hoc, la cual incluyó campos para el registro de los datos de las fichas clínicas de cada institución, y preguntas específicas para ser respondidas por el/la auxiliar de enfermería a cargo del hogar. En la 
ficha se registró: diagnóstico de fractura de cadera ocurrido en la institución y las variables edad, sexo, peso, talla, índice de masa corporal, tabaquismo, número de embarazos, histerectomia, uso de lentes, deambulación, y condición de usuario de benzodiazepinas (BDZ) y de otros medicamentos. También se consignó el registro de comorbilidades: hipertensión arterial (HTA), diabetes mellitus (DM), accidente vascular encefálico (AVE), epilepsia, daño orgánico cerebral, demencia, depresión, enfermedad bipolar, esquizofrenia, artrosis, cardiopatía, fracturas previas (excluyendo fractura de cadera) y fractura de cadera previa.

Finalmente, en los fracturados se registró también la edad al momento de la fractura, la presencia o no de acompañante, la estación del año, hora del día, lugar donde se produjo la fractura y la ocurrencia de secuelas.

El estudio piloto también permitió estimar la prevalencia de fractura de cadera que resultó ser del $10 \%$. Con este dato fue posible calcular un tamaño de muestra de 138 individuos, considerando un $95 \%$ de confianza y $5 \%$ de precisión. Se amplió este tamaño a un total de 200 individuos para permitir el estudio de asociaciones básicas de interés.

Se efectuó un análisis descriptivo que resume la información en medidas de tendencia central y dispersión. La comparación de datos continuos se realizó utilizando la prueba tstudent. La existencia de asociación entre fractura de cadera y diferentes factores de riesgo se exploró mediante la prueba de $\mathrm{x}^{2} \mathrm{y}$ análisis de varianza (Anova). Se calculó la razón de prevalencia (RP) considerando los principales factores de riesgo. Se utilizó el programa computacional Stata 7,0.

\section{RESULTADOS}

La edad de la muestra estudiada fluctuó entre 60 y 102 años y como era esperado, se encontró una mayor proporción de mujeres. Diferencias significativas entre ambos sexos $(\mathrm{p}<0,05)$ fueron constatadas en el promedio de edad, peso y talla (Tabla 1), pero no hubo diferencias en el índice de masa corporal (IMC). En cuanto a la frecuencia de comorbilidades, la hipertensión arterial presentó la mayor proporción con un $54,6 \%$, seguido de las enfermedades psiquiátricas con un $33,2 \%$.

El porcentaje de mujeres, la edad y la talla presentan diferencias $(\mathrm{p}<0,05)$ entre los Servicios de Salud, no así el peso promedio de los participantes el cual es similar (Tabla 2).

Se consignó un total de 19 casos de fractura de cadera en los 200 adultos mayores estudiados, lo que arroja una prevalencia de $9,5 \%$. No se observó diferencias significativas $(\mathrm{p}>0,05)$ por Servicio de Salud.

Entre las principales características asociadas a la fractura destaca que un $47,4 \%$ de los adultos mayores se encontraba sólo al momento del evento. Sólo 10 de los 19 casos tenían registrada la hora de ocurrencia, la cual fluctuó entre las 10:00 a.m. y las 20:00 pm. El lugar de caída fue registrado sólo en $2 / 3$ de los casos, siendo el más frecuente el baño $(n=5)$, seguido del dormitorio $(n=3)$, sala de estar $(n=2)$, jardín

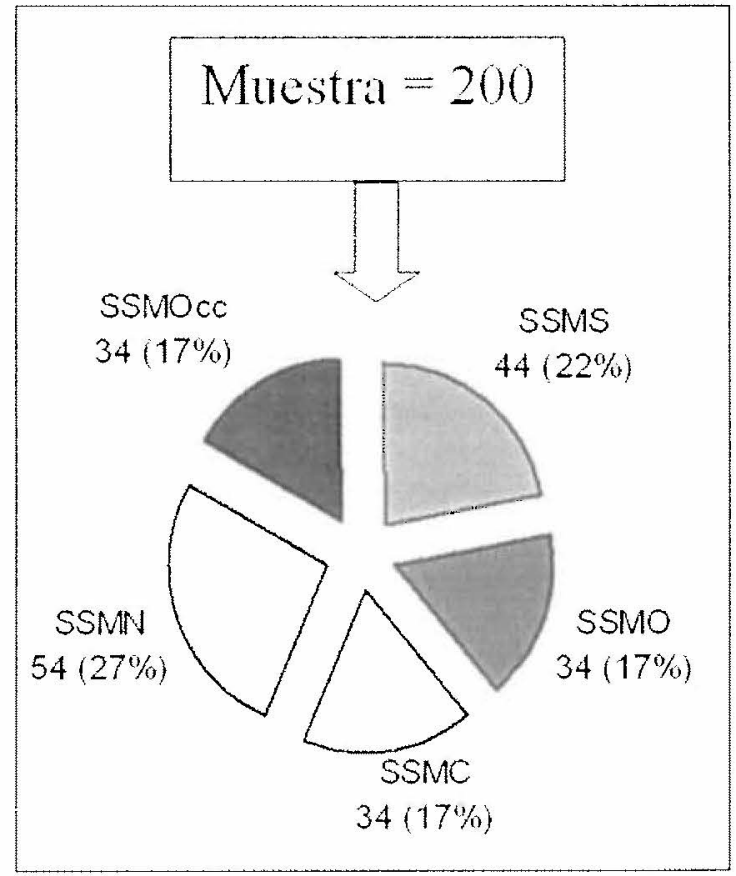

Figura 1. Selección de la muestra según distribución de los adultos mayores (A.M.) en los distintos Servicios de Salud. SSMS: Servicio de Salud Metropolitano Sur; SSMC: Servicio de Salud Metropolitano Centro; SSMO: Servicio de Salud Metropolitano Oriente; SSMOce: Servicio de Salud Metropolitano Occidente; SSMN: Servicio de Salud Metropolitano Norte. 
Tabla 1. Caracteristicas de la muestra estudiada

\begin{tabular}{lccr}
\hline Características & $\begin{array}{c}\text { Mujeres } \\
(\mathbf{n = 1 2 5})\end{array}$ & $\begin{array}{c}\text { Hombres } \\
(\mathbf{n}=\mathbf{7 5})\end{array}$ & $\begin{array}{c}\text { Total } \\
(\mathbf{n}=\mathbf{2 0 0})\end{array}$ \\
\hline \% del total & 62,5 & 37,5 & \\
Edad en años $(x+d s)$ & $81,9+8,8$ & $78,1+8,6^{*}$ & $80,4 \pm 8,9$ \\
Peso en $k g(x+d s)$ & $53,7+12,1$ & $58,9+10,2^{*}$ & $47,4 \pm 4,7$ \\
Talla en cm $(x+d s)$ & $150,1+8,1$ & $161,3+8,4^{*}$ & $154,2 \pm 9,9$ \\
IMC $(x+d s)$ & $23,9+5,1$ & $22,7+3,3^{* *}$ & $23,5 \pm 4,5$ \\
Prevalencia de fractura de cadera! & 11,2 & $6,67^{* * *}$ & 9,5 \\
\hline
\end{tabular}

* $p<0,05$ aplicando prueba $t$-student

$*^{* *} p>0,05$ aplicando prueba $t$-student

*** $\mathrm{p}>0,05$ aplicando prueba chi-cuadrado

? Prevalencia por cada 100 adultos mayor.

Tabla 2. Comparación de la muestra según Servicio de Salud

\begin{tabular}{lrrrrrr}
\hline Variable & SSMN & SSMS & SSMOce & SSMOr & SSMC & Valor p \\
\hline \% del total & 28,8 & 23,9 & 18,5 & 9,8 & 19 & \\
\% de mujeres & 49,1 & 50 & 67,7 & 55,6 & 100 & $\mathrm{p}<0,05^{1}$ \\
Edad en años $(x+\mathrm{ds})$ & $79,9 \pm 10,4$ & $79 \pm 8,2$ & $76,9 \pm 8,6$ & $82,9 \pm 8,0$ & $84,1 \pm 6,6$ & $\mathrm{p}<0,05^{2}$ \\
Peso en $\mathrm{kg}(\mathrm{x}+\mathrm{ds})$ & $58,2 \pm 10,6$ & $56,6 \pm 12,1$ & $54,5 \pm 12,1$ & $55,0 \pm 11,4$ & $51,8 \pm 12,2$ & $\mathrm{NS}^{2}$ \\
Talla en cm $(x+\mathrm{ds})$ & $1,58 \pm 8,7$ & $1,54 \pm 12,5$ & $1,54 \pm 10,5$ & $1,60 \pm 4,5$ & $1,47 \pm 6,0$ & $\mathrm{p}<0,05^{2}$ \\
Prevalencia de & 9,43 & 6,82 & 11,76 & 8,82 & 11,43 & $\mathrm{NS} 1$ \\
fractura de cadera & & & & & & \\
\hline
\end{tabular}

NS: No significativo

* Prevalencia por cada 100 adultos mayores

1 Aplicando prueba $\mathrm{X}^{2}$

2 Aplicando test de ANOVA

Tabla 3. Características de la población con fractura de cadera $v / s$ la que no tiene fractura

\begin{tabular}{lccr}
\hline Características & Fracturados & No Fracturados & Valor $\mathbf{p}$ \\
\hline Edad en años $(X+d s)$ & $82,3 \pm 9,7$ & $80,2 \pm 8,8$ & NS \\
Peso en $\mathrm{Kg}(X+\mathrm{ds})$ & $48,1 \pm 8,8$ & $56,6 \pm 11,6$ & $\mathrm{p}<0,05$ \\
Talla en $\mathrm{cm}(X+\mathrm{ds})$ & $150,3 \pm 9,6$ & $154,8 \pm 9,8$ & $\mathrm{p}=0,0529$ \\
IMC $(X+\mathrm{ds})$ & $21,5 \pm 2,1$ & $23,7 \pm 4,6$ & $\mathrm{p}<0,05$ \\
\hline
\end{tabular}

NS: no significativo

'Aplicando prueba t-student

$(\mathrm{n}=2)$ y el pasillo $(\mathrm{n}==1)$. No se observó diferencias significativas respecto la estación del año en que ocurren las fracturas.

Aparentemente el tamaño corporal opera como protector o al menos como predictor de menor riesgo de fractura, ya que los adultos mayores fracturados presentan menor peso e IMC $(\mathrm{p}<0,05)$ y menor estatura $(\mathrm{p}=0,05)$ (Tabla 3).
En cuanto a las consecuencias de la fractura de cadera, se observó que la autovalencia (capacidad de realizar por sí mismos las actividades de la vida diaria ${ }^{8}$ ), disminuyó en un $3,9 \%$ posterior al evento, siendo la postración la característica con mayor aumento $(2,5 \%)$ (Figura 2 ).

Al explorar factores de riesgo, se determinó que la probabilidad de fractura es mayor entre 
Tabla 4. Razón de prevalencia de factores de riesgo de fractura de cadera

\begin{tabular}{lccrc}
\hline Variable & \multicolumn{2}{c}{$\begin{array}{c}\text { Fractura de cadera } \\
\text { Prev. No exp. }\end{array}$} & $\begin{array}{c}\text { Razón de } \\
\text { Prevalencia }\end{array}$ & IC 95\% \\
\hline Sexol & 0,11 & 0,06 & 1,83 & $0,6-4,5$ \\
Fc. Previa & 0,18 & 0,07 & 2,57 & $1,1-5,8$ \\
Tabaquismo & 0,12 & 0,10 & 1,2 & $0,4-3,3$ \\
Histerectomia & 0,10 & 0,13 & 0,76 & $0,1-5,5$ \\
BZD, HTA, AVE 2 & 0,09 & 0,08 & 1,12 & $0,4-3,0$ \\
Presencia de enfermedades ${ }^{3}$ & 0,11 & 0,05 & 2,2 & $0,7-5,9$ \\
\hline
\end{tabular}

1 Sexo : se consideró como expuesto el sexo femenino.

2 Se consideró como expuesto si presenta al menos 1 de los siguientes antecedentes: uso de benzodiazepinas (BZD), hipertensión arterial (HTA), accidente vascular encefálico (AVE).

3 Se consideró como expuesto si presenta al menos 1 de las siguientes enfermedades: artrosis, enfermedad psiquiátrica, diabetes mellitus (DM), epilepsia, cardiopatías.

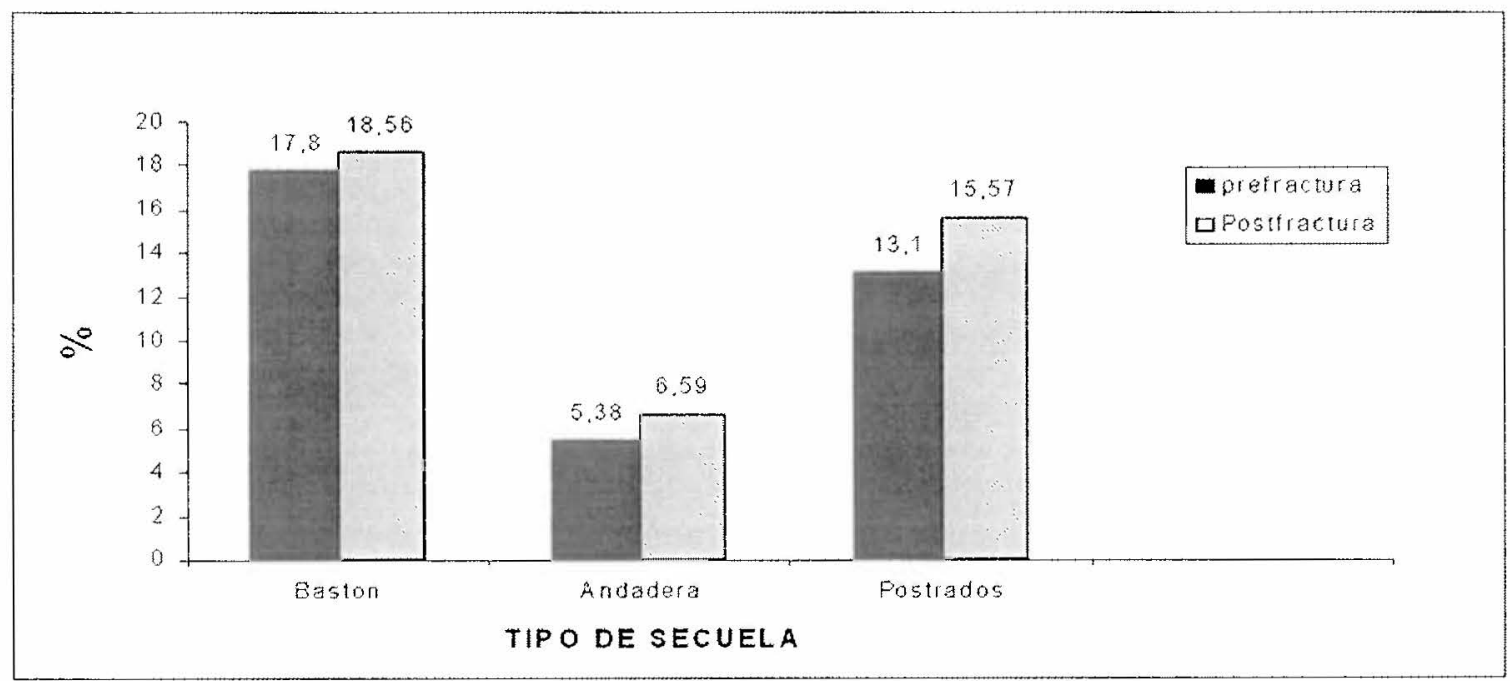

Figura 2. Grado de discapacidad de los AM previo y posterior a la fractura de cadera.

quienes han tenido una fractura previa (Tabla 4).

\section{DISCUSIÓN}

El estudio logró establecer la prevalencia de fractura de cadera en 9,5 por cada 100 adultos mayores institucionalizados. Este valor es similar al obtenido en un estudio italiano donde la prevalencia de fractura de cadera fue de 9 por cada 100 adultos mayores institucionalizados ${ }^{9}$, lo que reflejaría semejanzas en la estructura familiar, el origen caucásico de ambas poblaciones, estilos de vida y condiciones institucionales similares en ambos países ${ }^{10}$. Por el contrario, un estudio norteamericano revela una prevalencia de 4,7 por cada 100 mujeres mayores de 65 años ${ }^{11}$, diferencia que puede apuntar a las diferencias económico-sociales y en la infraestructura de las instituciones de adultos mayores.

El conocimiento de la prevalencia de fractura de cadera es relevante dado que esta patología implica un tratamiento quirúrgico no exento de complicaciones, que aumentan la morbimortalidad y disminuyen la calidad de $v^{\text {ida }}{ }^{12}$, generando una demanda en los servicios de salud, con los consiguientes costos para el Estado y la población afectada. Tomando en cuenta que existen pocos estudios nacionales al respecto, y que es una muestra representativa 
de los adultos mayores institucionalizados de la provincia de Santiago, la prevalencia obtenida adquiere mayor relevancia.

Una de las principales consecuencias de la fractura de cadera es el impacto que provoca en la autovalencia afectándola de diversas maneras, desde el uso de bastón hasta provocar el máximo grado de incapacidad - la postracióncaracterística que aumenta un 2,5\%, similar a lo descrito en la literatura ${ }^{13}$.

Se encontró un mayor IMC en quienes no han presentado esta patología, al igual que lo observado en otros estudios ${ }^{14-18}$, ya que el mayor peso corporal implica una mejor alimentación, mayor masa muscular y menor riesgo de presentar osteoporosis y caídas. Esto es coherente con estudios que demuestran que mejorando las condiciones nutricionales ${ }^{19-21}$ de los adultos mayores, e incorporando suplementos vitamínicos a su alimentación ${ }^{22-}$ ${ }^{24}$, se puede prevenir más efectivamente la fractura de cadera ${ }^{24}$.

Asimismo, se detectó que la historia de fracturas previas es un indicador de riesgo (RP 2,57; IC 95\% 1,1-5,8), información que puede ser de utilidad para la identificación de AM en riesgo en quienes extremar medidas preventivas.

En la caracterización del evento que determina la fractura destaca la ausencia de acompañante, la mayor ocurrencia en la sala de baño y en horas de mayor actividad, sin influencia estacional, lo que concuerda con lo encontrado en otros estudios ${ }^{25}$. Sin embargo, es llamativo el subregistro de la hora de ocurrencia, lo que lleva a especular que las caidas en horarios nocturnos son probablemente subnotificadas.

Como era de esperar la mayor proporción de fractura de cadera se encontró en población femenina, lo que se explica por una mayor esperanza de vida y un doble mecanismo de pérdida de masa ósea - envejecimiento e hipoestrogenismo - que condiciona a una mayor probabilidad de sufrir caídas ${ }^{26}$. En los distintos Servicios de Salud destaca la similar prevalencia de fractura de cadera a pesar de existir diferencias significativas en el porcentaje de mujeres, promedios de edad y talla.

Desde una perspectiva bioética se consideró la adecuada información a los directores de las instituciones seleccionadas, mediante una carta informativa y posterior visita, donde se solicitó la participación voluntaria en el estudio. No se solicitó consentimiento informado de cada participante, ya que la recolección de información se realizó a partir de las fichas clínicas, lo que a nuestro juicio no representa ningún procedimiento invasivo ni perjudicial. Por cierto se garantizó la confidencialidad de los datos obtenidos siendo utilizados sólo con fines de la presente investigación.

Posiblemente, la prevalencia derivada de este estudio está subestimada, ya que la muestra representa sólo a instituciones reglamentadas y acreditadas por los Servicios de Salud correspondientes, siendo en la actualidad 620 establecimientos a nivel nacional27. Quedan fuera las instituciones que funcionan de manera clandestina, que se estima en alrededor de 400 a nivel nacional ${ }^{27}$, las que albergan una población posiblemente con más factores de riesgo de fractura de cadera, derivados de eventuales fallas de la infraestructura y supervisión. La magnitud de dicha subestimación es difícil de establecer.

Este trabajo pone de manifiesto la falta de estudios referentes al adulto mayor y la necesidad de promoverlos dada la importancia que ha adquirido este grupo en nuestra población. Los resultados muestran un espacio para intervenciones que permitan corregir falencias en aspectos tan importantes como prevención de caídas, calidad de la alimentación y mejoramiento y supervisión de los mecanismos de seguridad y protección en Hogares de Adultos Mayores.

\section{REFERENCIAS}

1. www.ine.cl/cd2002/index php

2.- LOFMAN O, BERGLUND K, LARSSON L, TOSS $\mathrm{G}$. Changes in hip fracture epidemiology: redistribution between ages, genders and fracture types. Osteoporos Int 2002;13(1): 18-25

3.- DARGENT'-MOLINA P. Risk factors and prevention of fracture in the elderly. Curr Opin Rheumatol 1998; 10(4): 357-61. Review.

4.- SERRA J A, GARRIDO G, VIDAN M, MARANON E, BRANAS F, ORTIZ J. Epidemiology of hip fractures in the elderly in Spain. An Med Interna 2002; 19(8): 389-95.

5.- PUMARINOH, CONIRERAS L, KIRSCHBAUM 
A. Twelve-year trends of hip fracture rates in Chile. Is there a relationship between their increase and population aging?. Rev Méd Chile 1997; 125(8): 893-8.

6.- GIACONI J, VALDIVIA G, ARTEAGA E, PUMARINO H. La Osteoporosis en Chile: Fractura de cadera. Cuad Méd-Soc XXXV 4, 1994/3-9

7.- VALDIVIA G, GIACONI J, ARTEAGA E, PUMARINO $\mathrm{H}$, GAJARDO H, VILLAROEL $\mathrm{L}$. Fractura de la cadera: Estudio de casos y controles en la RM I. Rev Méd Chile 1996; 124: $189-97$.

8. POLÍTICA SOCIAL PARA EL ADULTOMAYOR, aprobada por el Comité de Ministros para el Adulto Mayor en sesión constitutiva del 06 de mayo de 2004.

9.- MAGGIO D, UBALDI E, SIMONELLI G, CENCI S, PEDONE C, CHERUBINI A. Hip fracture in nursing homes: an Italian study on prevalence, latency, risk factors, and impact on mobility. Calcif Tissue Int 2001; 68(6): 337-41.

10.- www.italica.rai.it/principali/lingua/culture/ famiglia.htm

11.- HOCHBERG M, WILLIAMSON J, SKINNER E, GURALNIK J, KASPER J, FRIED L. The prevalence and impact of self-reported hip fracture in elderly community-dwelling women: the Women's Health and Aging Study. Osteoporos Int 1998; 8(4): 385-9.

12.- MAROTTOLIR, BERKMAN L, LEO-SUMMERS L, COONEY L M Jr. Predictors of mortality and institutionalization after hip fracture: the New Haven EPESE cohort. Established Populations for Epidemiologic Studies of the Elderly. Am J Public Health. 1994; 84(11): 1807-12.

13.- MAGAZINER J, FREDMAN L, HAWKES W, HEBEL J, ZIMMERMAN S, ORWIG $\mathrm{L}$ et al. Changes in functional status attributable to hip fracture: a comparison of hip fracture patients to community-dwelling aged. Am J Epidemiol 2003; 157(11): 1023-31.

14.- LANGLOIS J, VISSER M, DAVIDOVIC L, MAGGI S, LI G, HARRIS T. Hip fracture risk in older white men is associated with change in body weight from age 50 years to old age. Arch Intern Med 1998; 11;158(9): 990-6.

15.- LANGLOIS J, HARRIS T, LOOKER A, MADANS $J$. Weight change between age 50 years and old age is associated with risk of hip fracture in white women aged 67 years and older. Arch Intern Med 1996; 13; 156(9): 989-94.

16.- LANGLOIS J, MUSSOLINO M, VISSER $M$,
LOOKER A, HARRIS T, MADANS J. Weight loss from maximum body weight among middle-aged and older white women and the risk of hip fracture: the NHANES 1 epidemiologic follow-up study. Osteoporos Int 2001; 12(9): 763-8.

17. - FARAHMAND B, MICHAELSSON K, BARON J, PERSSON P, LJUNGHALL $S$. Body size and hip fracture risk. Swedish Hip Fracture Study Group. Epidemiology 2000; 11(2): 214-9.

18.- HUANG Z, HIMES J, MCGOVERN P. Nutrition and subsequent hip fracture risk among a national cohort of white women. Am J Epidemiol 1996 15; 144(2): 124-34.

19.- MUNGER R, CERHAN J, CHIU B. Prospective study of dietary protein intake and risk of hip fracture in postmenopausal women. Am J Clin Nutr 1999; 69(1): 147-52.

20.- WENGREEN H, MUNGER R, CUTLER D, CORCORAN C, ZHANG J, SASSANON. Dietary protein intake and risk of osteoporotic hip fracture in elderly residents of Utah. J Bone Miner Res $2004 ; 19(4): 537-45$.

21.- LOOKER A, HARRIS T, MADANS J, SEMPOS C. Dietary calcium and hip fracture risk: the NHANES I Epidemiologic Follow-Up Study. Osteoporos Int 1993; 3(4): 177-84.

22.- FESKANICH D, WILLETT W, COLDITZ G. Prospective study of dietary protein intake and risk of hip fracture in postmenopausal women. Am J Clin Nutr 1999; 69(1): 147-52.

23.- FESKANICH D, WEBER P, WILLETT $W$, ROCKETT H, BOOTH S, COLDITZ G.Vitamin K intake and hip fractures in women: a prospective study. Am J Clin Nutr 1999; 69(1): 74-9.

24.- LILLIU H, PAMPHILE R, CHAPUY M, SCHULTEN J, ARLOT M, MEUNIER P. Calciumvitamin D3 supplementation is cost-effective in hip fractures prevention. Maturitas $2003 ; 25 ; 44(4)$ : $299-305$

25.- AHARONOFF G, DENNIS M, ELSHINAWY A, ZUCKERMAN J, KOVAL K. Circumstances of falls causing hip fractures in the elderly. Clin Orthop 1998; (348): 10-4.

26.- YATES J, BARRETT-CONNOR E, BARLAS S, CHEN Y, MILLER P, SIRIS E. Rapid loss of hip fracture protection after estrogen cessation: evidence from the National Osteoporosis Risk Assessment. Obstet Gynecol 2004; 103(3): 440-6.

27. http://www. senama.cl/amayor/pags/prensa/ amplianoticia.asp? noticia $=84$.

Usted puede comentar éste y otros artículos publicados en la Revista Chilena de Salud Pública, enviando un correo electrónico a revistasp@med.uchile.cl 